\title{
Convergence Analysis of the Finite Difference Solution for the Nonlinear Klein-Gordon Equation
}

\author{
Ekhlass S. Al-Rawi \\ drekhlass-alrawi@uomosul.edu.iq \\ College of computers \& Mathematical Sciences \\ University of Mosul, Iraq
}

Received on: 22/03/2007

Accepted on: 28/06/2007

This paper is devoted to drive the matrix algebraic equation for the onedimensional nonlinear Klein-Gordon equation which is obtained from using the implicit finite difference method. The convergence analysis of the solution is discussed. Numerical computations are conducted and the solutions are stable and convergent when the sine function is used as an initial condition.

Keywords: Implicit finite difference method; Nonlinear Klein-Gordon equation; convergence analysis.

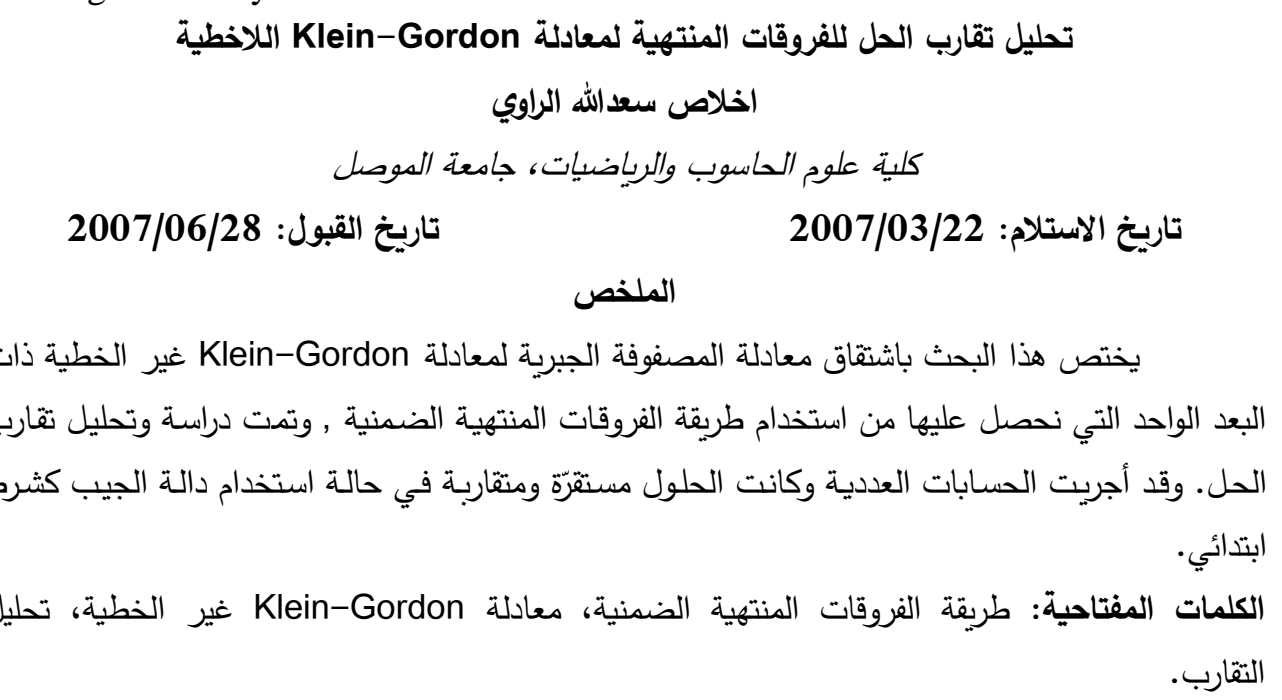

\section{1-Introduction}

Let us consider the following nonlinear Klein-Gordon equation (NKG) $[2,4]$

$$
\frac{\partial^{2} \psi}{\partial t^{2}}-\Delta \psi+c^{2} \psi-|\psi|^{p-2} \psi=0
$$

where $\psi=\psi(x, t)$ is a function from $(0, L) \times(0, \infty) \subset R \times R$ to $R$, and $c \in R$

with Dirichlet boundary conditions

$$
\psi(t, 0)=0=\psi(t, L), \quad 0 \leq t<\infty
$$

and initial conditions 


$$
\left.\psi\right|_{t=0}=u_{0},\left.\quad \psi_{t}\right|_{t=0}=v_{0}, 0 \leq x \leq L
$$

let $\mathrm{p}=4$ eq.(1) becomes

$$
\frac{\partial^{2} \psi}{\partial t^{2}}-\Delta \psi+c^{2} \psi-|\psi|^{2} \psi=0
$$

The relativistic wave equation of the motion of a free particle with zero spin, found by physicists O. Klein and V. Gordon is called KleinGordon equation (KGE)[1]. The Klein-Gordon equation is one of the nonlinear extensions of the wave equation. For example, such an equation describes the vibration of a string that lies on an elastic foundation with nonlinear elastic forces. The Klein-Gordon equation, and especially the SinGordon equation, is often encountered in physics [3]. From equation (1) it is possible to develop the theory of electrically charged fields and study the interaction of $\psi$ with an assigned electromagnetic field. On the other hand, it is also possible to study the interaction of $\psi$ with its own electromagnetic field, which is not assigned but is an unknown of the problem. In the last years a wide interest was born about solitary waves of eq.(1), i.e. solutions of the form

$$
\psi(x, t)=u(x) e^{i w t}
$$

where $\mathrm{u}$ is a real bounded function and $w \in R[4]$.

Jean-Philippe Nicolas [5] studied the nonlinear Klein-Gordon equation outside a slow kerr black hole and solved the global Cauchy problem for large data with minimum regularity and also proved the existence of smooth profiles and Summerfield radiation conditions. Dimitri Mugnai [4] established the existence of infinitely many nontrivial radially symmetric solitary waves for the nonlinear Klein-Gordon equation, coupled with a Born-Infeld type equation under general assumptions. Bektas, Bulut and Ergut [1] obtained the Klein-Gordon equation(KGE) in the Galilean space and applied Adomain Decomposition method to (KGE), the advantage of the decomposition methodology displays a fast convergence of the solutions. The decomposition method provides a reliable technique that requires less work if compared with the traditional techniques .Jean-Marc Delort and Jeremie Szefel [2] devoted to the proof of almost global existence results for Klein-Gordon equations on compact resolution hypersurfaces with non-Hamiltonian nonlinearities when the data are smooth, small and radial and used the method combines normal forms with the fact that the eigenvalues associated with radial eigenfunctions of the Laplacian on such manifolds are simple and satisfy convenient asymptotic expansions.

In this paper, we will derive the matrix equation for the onedimensional Klein-Gordon equation which is obtained from using finite 
difference method by using the implicit time discretization method, then we will prove the existence of the solution of the matrix equation, and give two examples for the Klein-Gordon equation in order to show the convergence analysis for the numerical results.

Lemma (1): The nonlinear Klein-Gordon equation (1) has a unique solution $\psi$ continuous and bounded and also $\partial_{t} \psi$ is continuous and bounded [2].

\section{2-Derivation of the matrix equation using the finite difference method}

We introduce a uniform grid by defining the following discrete set of points in the $\mathrm{x}, \mathrm{t}$ plane:

$$
\begin{aligned}
\mathrm{x}_{\mathrm{i}} & =\mathrm{ih}, & & \mathrm{i}=0,1, \ldots, \mathrm{n}-1, \mathrm{n} \\
\mathrm{t}_{\mathrm{j}} & =\mathrm{jk}, & & \mathrm{j}=0,1, \ldots, \mathrm{m}-1, \mathrm{~m}
\end{aligned}
$$

The discretized solution of equation (2), by using the implicit finite difference method is

$$
\begin{aligned}
& \frac{1}{k^{2}}\left(\psi^{j}\left(x_{i}\right)-2 \psi^{j-1}\left(x_{i}\right)+\psi^{j-2}\left(x_{i}\right)\right)-\frac{1}{h^{2}}\left(\psi^{j}\left(x_{i+1}\right)-2 \psi^{j}\left(x_{i}\right)+\psi^{j}\left(x_{i-1}\right)\right) \\
& +c^{2} \psi^{j}\left(x_{i}\right)-\left|\psi^{j}\left(x_{i}\right)\right|^{2} \psi^{j}\left(x_{i}\right)=0
\end{aligned}
$$

Divide the interval $(0, \mathrm{~L})$ into $\mathrm{n}$ subintervals $\left(x_{i-1}, x_{i}\right)$ with length $\frac{1}{n}$, $\mathrm{i}=1,2, \ldots, \mathrm{n}, 0=\mathrm{x}_{0}<\mathrm{x}_{1}<\ldots<\mathrm{x}_{\mathrm{n}-1}<\mathrm{x}_{\mathrm{n}}=\mathrm{L}$, and multiply equation (3) by $\mathrm{k}^{2}$, we get the following equation

$$
\begin{aligned}
& \psi^{j}\left(x_{i}\right)-2 \psi^{j-1}\left(x_{i}\right)+\psi^{j-2}\left(x_{i}\right)-\frac{k^{2}}{h^{2}}\left(\psi^{j}\left(x_{i+1}\right)-2 \psi^{j}\left(x_{i}\right)+\psi^{j}\left(x_{i-1}\right)\right) \\
& +c^{2} k^{2} \psi^{j}\left(x_{i}\right)-k^{2}\left|\psi^{j}\left(x_{i}\right)\right|^{2} \psi^{j}\left(x_{i}\right)=0
\end{aligned}
$$

after arranging the above equation in terms of $\psi^{j}\left(x_{i-1}\right), \psi^{j}\left(x_{i}\right)$ and $\psi^{j}\left(x_{i+1}\right)$, we get

$$
\begin{aligned}
& -\frac{k^{2}}{h^{2}} \psi^{j}\left(x_{i-1}\right)+\left(1+\frac{2 k^{2}}{h^{2}}+c^{2} k^{2}-k^{2}\left|\psi^{j}\left(x_{i}\right)\right|^{2}\right) \psi^{j}\left(x_{i}\right)-\frac{k^{2}}{h^{2}} \psi^{j}\left(x_{i+1}\right)=2 \psi^{j-1}\left(x_{i}\right)-\psi^{j-2}\left(x_{i}\right) \\
& A\left(\psi^{j}\left(x_{i}\right)\right) \psi^{j}\left(x_{i-1}\right)+B\left(\psi^{j}\left(x_{i}\right)\right) \psi^{j}\left(x_{i}\right)+A\left(\psi^{j}\left(x_{i}\right)\right) \psi^{j}\left(x_{i+1}\right)=F\left(\psi^{j-1}\left(x_{i}\right)\right) \\
& \text { for } \mathrm{i}=1,2, \ldots, \mathrm{n}-1, \text { where } \\
& A\left(\psi^{j}\left(x_{i}\right)\right)=-\frac{k^{2}}{h^{2}} \\
& B\left(\psi^{j}\left(x_{i}\right)\right)=1+\frac{2 k^{2}}{h^{2}}+c^{2} k^{2}-k^{2}\left|\psi^{j}\left(x_{i}\right)\right|^{2} \\
& F\left(\psi^{j-1}\left(x_{i}\right)\right)=2 \psi^{j-1}\left(x_{i}\right)-\psi^{j-2}\left(x_{i}\right)
\end{aligned}
$$


Note that the definitions of $B\left(\psi^{j}\left(x_{i}\right)\right)$ depend on $\psi^{j}\left(x_{i}\right)$ and $F\left(\psi^{j-1}\left(x_{i}\right)\right)$ depend on $\psi^{j-1}\left(x_{i}\right)$ and $\psi^{j-2}\left(x_{i}\right)$. Equation (4) now becomes in following algebraic matrix equation.:

$$
\mathrm{C}\left(\psi^{j}\right)\left\{\psi^{j}\right\}=\left\{\mathrm{F}\left(\psi^{j-1}\right)\right\}
$$

where $\square \square \mathrm{C}\left(\psi^{j}\right)=\left\{C_{l, s}\left(\psi^{j}\left(x_{i}\right)\right)\right\}_{(n-1) \times(n-1)}$ is the tri-diagonal matrix.

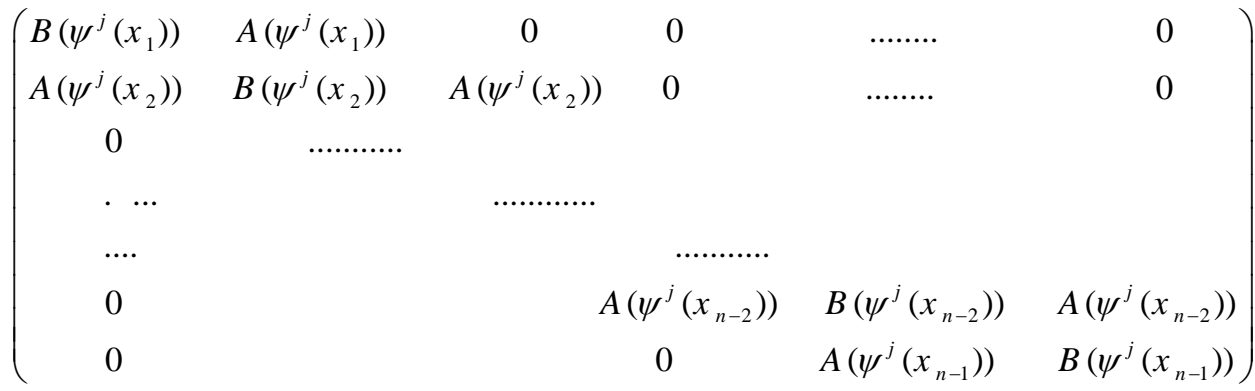

$$
\begin{aligned}
& \left\{\mathrm{F}\left(\psi^{j-1}\right)\right\}=\left\{\mathrm{F}\left(\psi^{\mathrm{j}-1}\left(\mathrm{x}_{1}\right)\right), \mathrm{F}\left(\psi^{\mathrm{j}-1}\left(\mathrm{x}_{2}\right)\right), \ldots ., \mathrm{F}\left(\psi^{\mathrm{j}-1}\left(\mathrm{x}_{\mathrm{n}-1}\right)\right)\right\}^{\mathrm{T}}
\end{aligned}
$$

Since $A\left(\psi^{j}\left(x_{i}\right)\right)$ is a constant then the matrix $\square \square\left(\psi^{j}\right)$ is symmetric.

In each time step j€ $\square \mathrm{N}$, we assume that $\square \mathrm{C}\left(\psi_{i}^{j}\right)=\mathrm{C}\left(\psi_{i-1}^{j}\right), \psi_{0}^{j}=\psi^{j-1}$ for $\mathrm{i} \in \mathrm{N}$ which allows the use of an iterative solution method to compute $\psi^{\mathrm{j}}$. we stop when the norm of the difference of $\psi_{i}^{j}$ and $\psi_{i-1}^{j}$ is sufficiently small. This method is called the direct iterative method.

\section{3- Existence of the Finite Difference Solution}

we now prove the existence of the solution of the matrix equation.

Theorem (1): The solution of the matrix equation

$$
\square \mathrm{C}\left(\psi^{j}\right)\left\{\psi^{j}\right\}=\left\{\mathrm{F}\left(\psi^{j-1}\right)\right\} \text { exists. }
$$

Proof: Let $\mathrm{j} \in \mathrm{N}$ be fixed. Consider the following iteration of the matrix equation

$$
\mathrm{C}\left(\psi_{i-1}^{j}\right)\left\{\psi_{i}^{j}\right\}=\left\{\mathrm{F}\left(\psi^{j-1}\right)\right\} \quad, \quad \forall i \in \mathrm{N}
$$

where $\psi_{0}^{j}=\psi^{j-1}$. By subtracting the equation(5) from

$\mathrm{C}\left(\psi_{i}^{j}\right)\left\{\psi_{i+1}^{j}\right\}=\left\{\mathrm{F}\left(\psi^{j-1}\right)\right\} \quad$, we have

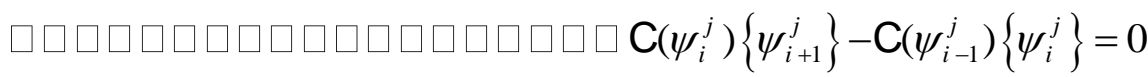

$$
\mathrm{C}\left(\psi_{i}^{j}\right)\left\{\psi_{i+1}^{j}-\psi_{i}^{j}\right\}=\left(\mathrm{C}\left(\psi_{i-1}^{j}\right)-\mathrm{C}\left(\psi_{i}^{j}\right)\right)\left\{\psi_{i}^{j}\right\}
$$


from the right hand side of eq.(6), the $\mathrm{k}^{\text {th }}$ element is

$$
\sum_{s=1}^{n-1}\left\{C_{k, s}\left(\psi_{\mathrm{i}-1}^{\mathrm{j}}\left(x_{k}\right)\right)-C_{k, s}\left(\psi_{\mathrm{i}}^{\mathrm{j}}\left(x_{k}\right)\right)\right\} \cdot \psi_{\mathrm{i}}^{\mathrm{j}}\left(x_{s}\right)
$$

By the Mean Value theorem, it becomes

$$
\sum_{s=1}^{n-1} \sum_{l=1}^{n-1}\left[C_{k, s}\left(\psi_{\mathrm{i} *}^{\mathrm{j}}\left(x_{k}\right)\right)\right]_{x_{l}} \cdot\left(\psi_{\mathrm{i}-1}^{\mathrm{j}}\left(x_{l}\right)-\psi_{\mathrm{i}}^{\mathrm{j}}\left(x_{l}\right)\right) \cdot \psi_{\mathrm{i}}^{\mathrm{j}}\left(x_{s}\right)
$$

where the value of $\psi_{i *}^{j}\left(x_{k}\right)$ is between $\psi_{i-1}^{j}\left(x_{k}\right)$ and $\psi_{i}^{j}\left(x_{k}\right)$, and $\left[C_{k, s}\left(\psi_{\mathrm{i} *}^{\mathrm{j}}\left(x_{k}\right)\right)\right]_{x_{l}}$ represents the partial derivatives of $C_{k, s}\left(\psi_{\mathrm{i} *}^{\mathrm{j}}\left(x_{k}\right)\right)$ with respect to $\psi_{i *}^{j}\left(x_{l}\right)$.

Now, the right hand side of eq.(6) becomes

$\sum_{l=1}^{n-1} \sum_{s=1}^{n-1} \psi_{\mathrm{i}}^{\mathrm{j}}\left(x_{s}\right) \cdot\left[C_{k, s}\left(\psi_{\mathrm{i} *}^{\mathrm{j}}\left(x_{k}\right)\right)\right]_{x_{l}} \cdot\left(\psi_{\mathrm{i}-1}^{\mathrm{j}}\left(x_{l}\right)-\psi_{\mathrm{i}}^{\mathrm{j}}\left(x_{l}\right)\right)$

$$
=\square \mathrm{D}\left(\psi_{i *}^{j}\right)\left\{\psi_{i-1}^{j}-\psi_{i}^{j}\right\}
$$

where $\mathrm{D}\left(\psi_{i *}^{j}\right)$ is the following matrix

$$
\left(\begin{array}{ccccc}
\sum_{s=1}^{n-1} \psi_{\mathrm{i}}^{\mathrm{j}}\left(x_{s}\right) & \cdot\left[C_{1, s}\left(\psi_{\mathrm{i} *}^{\mathrm{j}}\left(x_{1}\right)\right)\right]_{x_{1}} & \ldots & \sum_{s=1}^{n-1} \psi_{\mathrm{i}}^{\mathrm{j}}\left(x_{s}\right) & \cdot\left[C_{1, s}\left(\psi_{\mathrm{i} *}^{\mathrm{j}}\left(x_{1}\right)\right)\right]_{x_{n-1}} \\
\sum_{s=1}^{n-1} \psi_{\mathrm{i}}^{\mathrm{j}}\left(x_{s}\right) \cdot\left[C_{2, s}\left(\psi_{\mathrm{i} *}^{\mathrm{j}}\left(x_{2}\right)\right)\right]_{x_{1}} & \ldots & \sum_{s=1}^{n-1} \psi_{\mathrm{i}}^{\mathrm{j}}\left(x_{s}\right) & \cdot\left[C_{2, s}\left(\psi_{\mathrm{i} *}^{\mathrm{j}}\left(x_{2}\right)\right)\right]_{x_{n-1}} \\
\ldots & \ldots & \ldots \\
\sum_{s=1}^{n-1} \psi_{\mathrm{i}}^{\mathrm{j}}\left(x_{s}\right) \cdot\left[C_{n-2, s}\left(\psi_{\mathrm{i} *}^{\mathrm{j}}\left(x_{n-2}\right)\right)\right]_{x_{1}} & \ldots & \sum_{s=1}^{n-1} \psi_{\mathrm{i}}^{\mathrm{j}}\left(x_{s}\right) & \cdot\left[C_{n-2, s}\left(\psi_{\mathrm{i} *}^{\mathrm{j}}\left(x_{n-2}\right)\right)\right]_{x_{n-1}} \\
\sum_{s=1}^{n-1} \psi_{\mathrm{i}}^{\mathrm{j}}\left(x_{s}\right) \cdot\left[C_{n-1, s}\left(\psi_{\mathrm{i} *}^{\mathrm{j}}\left(x_{n-1}\right)\right)\right]_{x_{1}} & \ldots & \sum_{s=1}^{n-1} \psi_{\mathrm{i}}^{\mathrm{j}}\left(x_{s}\right) & \cdot\left[C_{n-1, s}\left(\psi_{\mathrm{i} *}^{\mathrm{j}}\left(x_{n-1}\right)\right)\right]_{x_{n-1}}
\end{array}\right)
$$

since $C_{1, s}\left(\psi_{\mathrm{i} *}^{\mathrm{j}}\left(x_{1}\right)\right), \mathrm{s}=1,2, \ldots, \mathrm{n}-1$ contains only $\psi_{\mathrm{i} *}^{\mathrm{j}}\left(x_{1}\right)$ then

$D_{1, l}\left(\psi_{i *}^{\mathrm{j}}\right)=\sum_{s=1}^{n-1} \psi_{\mathrm{i}}^{\mathrm{j}}\left(x_{s}\right) \cdot\left[C_{1, s}\left(\psi_{\mathrm{i} *}^{\mathrm{j}}\left(x_{1}\right)\right)\right]_{x_{l}}=0 \quad$, if $\quad l \neq 1$ 


$$
\begin{aligned}
& \text { when } l=1 \\
& \begin{aligned}
D_{1,1}\left(\psi_{i *}^{\mathrm{j}}\right)= & \sum_{s=1}^{n-1} \psi_{\mathrm{i}}^{\mathrm{j}}\left(x_{s}\right) \cdot\left[C_{1, s}\left(\psi_{\mathrm{i} *}^{\mathrm{j}}\left(x_{1}\right)\right)\right]_{x_{1}} \\
& =\psi_{\mathrm{i}}^{\mathrm{j}}\left(x_{1}\right) \cdot\left[C_{1,1}\left(\psi_{\mathrm{i} *}^{\mathrm{j}}\left(x_{1}\right)\right)\right]_{x_{1}}+\psi_{\mathrm{i}}^{\mathrm{j}}\left(x_{2}\right) \cdot\left[C_{1,2}\left(\psi_{\mathrm{i} *}^{\mathrm{j}}\left(x_{1}\right)\right)\right]_{x_{1}} \\
& =\psi_{\mathrm{i}}^{\mathrm{j}}\left(x_{1}\right)\left[1+\frac{2 k^{2}}{h^{2}}+c^{2} k^{2}-k^{2}\left|\psi_{i *}^{\mathrm{j}}\left(x_{1}\right)\right|^{2}\right]+\psi_{\mathrm{i}}^{\mathrm{j}}\left(x_{2}\right)\left[\frac{-k^{2}}{h^{2}}\right]_{x_{1}} \\
& =\psi_{\mathrm{i}}^{\mathrm{j}}\left(x_{1}\right)\left[-2 k^{2}\left|\psi_{i *}^{\mathrm{j}}\left(x_{1}\right)\right|\right]+0 \\
& \leq 2 k^{2}\left|\psi_{\mathrm{i} *}^{\mathrm{j}}\left(x_{1}\right)\right| \cdot\left|\psi_{i}^{\mathrm{j}}\left(x_{1}\right)\right|
\end{aligned}
\end{aligned}
$$

since $\mathrm{k}^{2}$ is sufficiently small and $\psi^{\mathrm{j}}$ is bounded from Lemma(1), then $D_{1,1}\left(\psi_{i *}^{j}\right)$ in equation (7) is bounded by a small number. Therefore, all the elements in the first row of the matrix $\mathrm{D}\left(\psi_{i *}^{j}\right)$ are zero except for the first element, and the first element is sufficiently small.

Similarly $C_{n-1, s}\left(\psi_{\mathrm{i} *}^{\mathrm{j}}\left(x_{n-1}\right)\right), s=1,2, \ldots, n-1$ only contains $\psi_{\mathrm{i} *}^{\mathrm{j}}\left(x_{n-1}\right)$ which implies if $l \neq n-1, \quad D_{n-1, l}\left(\psi_{i *}^{j}\right)=0$

when $l=n-1$

$$
\begin{aligned}
D_{n-1, n-1}\left(\psi_{i *}^{\mathrm{j}}\right) & =\psi_{\mathrm{i}}^{\mathrm{j}}\left(x_{n-2}\right) \cdot\left[C_{n-1, n-2}\left(\psi_{\mathrm{i} *}^{\mathrm{j}}\left(x_{n-1}\right)\right)\right]_{x_{n-1}}+\psi_{\mathrm{i}}^{\mathrm{j}}\left(x_{n-1}\right) \cdot\left[C_{n-1, n-1}\left(\psi_{\mathrm{i} *}^{\mathrm{j}}\left(x_{n-1}\right)\right)\right]_{x_{n-1}} \\
& =\psi_{\mathrm{i}}^{\mathrm{j}}\left(x_{n-1}\right)\left[-2 k^{2}\left|\psi_{i *}^{\mathrm{j}}\left(x_{n-1}\right)\right|\right] \\
& \leq 2 k^{2}\left|\psi_{\mathrm{i} *}^{\mathrm{j}}\left(x_{n-1}\right)\right| \cdot\left|\psi_{i}^{\mathrm{j}}\left(x_{n-1}\right)\right| \\
& \leq \varepsilon_{1}
\end{aligned}
$$

for some $\varepsilon_{1}<<1$. Thus all the elements in the $(\mathrm{n}-1)^{\text {th }}$ row of the matrix $\square \mathrm{D}\left(\psi_{i *}^{j}\right)$ are zero except for the $(\mathrm{n}-1)^{\mathrm{th}}$ element, and $(\mathrm{n}-1)^{\text {th }}$ element is bounded by a small number.

Since only $\psi_{\mathrm{i} *}^{\mathrm{j}}\left(x_{k}\right)$ is in the $C_{k, s}\left(\psi_{\mathrm{i} *}^{\mathrm{j}}\left(x_{k}\right)\right), \quad k \neq 1, n-1, \quad s=1,2, \ldots, n-1$

$$
D_{k, l}\left(\psi_{i *}^{j}\right)=0 \quad, \quad \text { if } \quad l \neq k
$$

when $l=k$

$$
\begin{aligned}
D_{k, k}\left(\psi_{i *}^{j}\right) & =\psi_{\mathrm{i}}^{\mathrm{j}}\left(x_{k}\right) \cdot\left[C_{k, k}\left(\psi_{\mathrm{i} *}^{\mathrm{j}}\left(x_{k}\right)\right)\right]_{x_{k}} \\
& =\psi_{\mathrm{i}}^{\mathrm{j}}\left(x_{k}\right)\left[-2 k^{2}\left|\psi_{i *}^{\mathrm{j}}\left(x_{k}\right)\right|\right] \\
& \leq 2 k^{2}\left|\psi_{\mathrm{i} *}^{\mathrm{j}}\left(x_{k}\right)\right| \cdot\left|\psi_{i}^{\mathrm{j}}\left(x_{k}\right)\right| \\
& \leq \varepsilon_{2}
\end{aligned}
$$

for some $\varepsilon_{2}<<1$. 
Therefore, the matrix $\mathrm{D}\left(\psi_{i *}^{j}\right)$ is of the following form:

$$
\left(\begin{array}{ccccccc}
D_{1,1}\left(\psi_{i *}^{j}\right) & 0 & 0 & 0 & \ldots \ldots & 0 & 0 \\
0 & D_{2,2}\left(\psi_{i *}^{j}\right) & 0 & 0 & \ldots \ldots & 0 & 0 \\
0 & 0 & D_{3,3}\left(\psi_{i *}^{j}\right) & 0 & \ldots \ldots & 0 & 0 \\
\cdot & \cdot & \cdot & & & & \cdot \\
\cdot & \cdot & \cdot & & & & \\
\cdot & \cdot & \cdot & & & & \\
0 & 0 & 0 & 0 & \ldots \ldots & 0 & D_{n-1, n-1}\left(\psi_{i *}^{j}\right)
\end{array}\right)
$$

where the diagonal elements are sufficiently small. Thus, the norm of the matrix $\square \mathrm{D}\left(\psi_{i *}^{j}\right)$, which is defined by $\sup _{x \in R^{n-1}}\left\|\mathrm{D}\left(\psi_{i *}^{j}(x)\right)\right\|_{L^{2}}$ is small and bounded.

Now, it remains to show that $\mathrm{C}\left(\psi_{i}^{j}\right)$ is invertible and bounded away from zero. Consider the matrix $\mathrm{C}\left(\psi_{i}^{j}\right)$ as the sum of three matrices $\mathrm{J}, \mathrm{K}$, and $\mathrm{L}\left(\psi_{i}^{j}\right)$, where these matrices are:

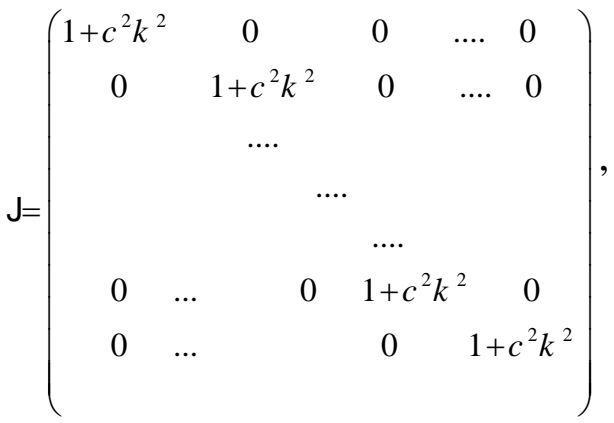

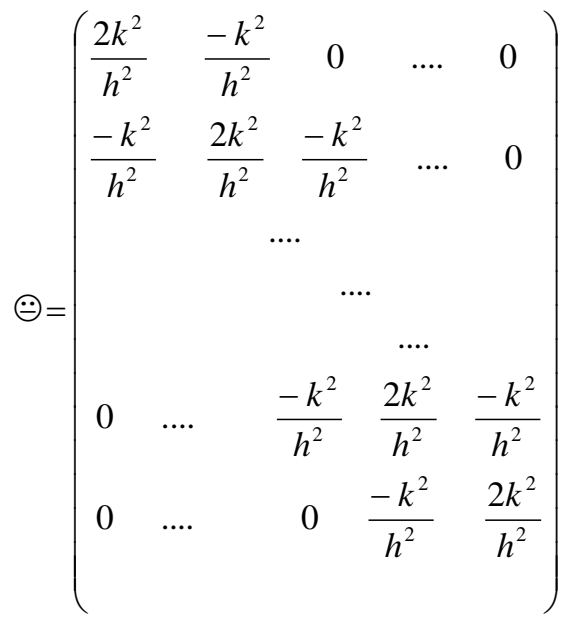


and $\mathrm{L}\left(\psi_{i}^{j}\right)$ is the following diagonal matrix

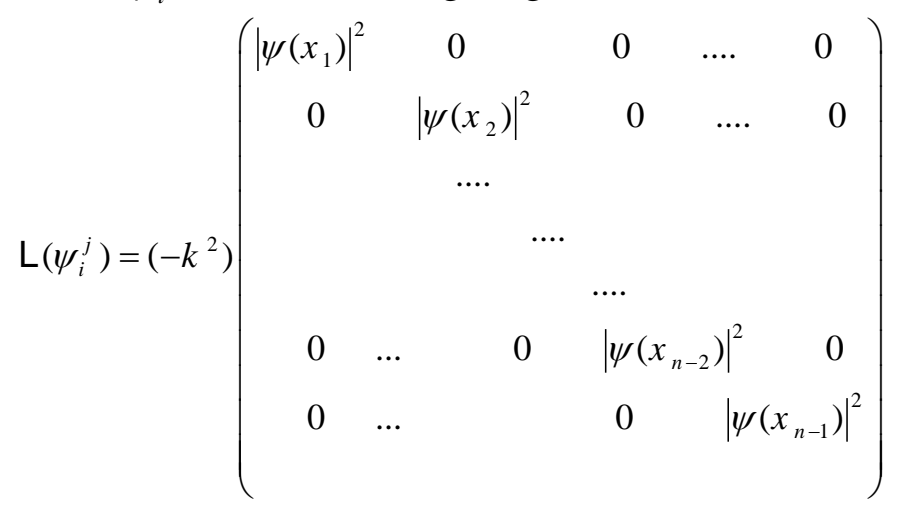
positive

The matrix $:-$ is positive definite since eigenvalues of the matrix are

$$
\left|\lambda_{i}-k_{i i}\right| \leq \sum_{j \neq i}\left|k_{i j}\right|
$$

where $k_{i i}=2 \frac{k^{2}}{h^{2}}$, and $\sum_{j \neq i}\left|k_{i j}\right| \geq 2 \frac{k^{2}}{h^{2}}$. Thus the matrix $\mathrm{C}\left(\psi_{i}^{j}\right)$ is positive definite and bounded away from zero since

$$
\begin{aligned}
\xi^{T} \cdot \mathrm{C} \cdot \xi & =\left(1+c^{2} k^{2}\right) \cdot|\xi|^{2}+\xi^{T} \cdot \mathrm{K} \cdot \xi+\xi^{T} \cdot \mathrm{L}\left(\psi_{i}^{j}\right) \cdot \xi \\
& \geq\left(1+c^{2} k^{2}\right) \cdot|\xi|^{2}-k^{2}\left|\psi_{i}\right|^{2} \cdot|\xi|^{2} \\
& =\left(1+c^{2} k^{2}-k^{2}\left|\psi_{i}\right|^{2}\right) \cdot|\xi|^{2} \\
& >\frac{1}{2}|\xi|^{2} \\
& >0
\end{aligned}
$$

The proof is complete.

\section{4- Numerical computations}

In this section, we have solved two different examples by using the finite difference method (Implicit method).

Example (1): we consider a linear KGE of the form [6]

$$
\partial_{t}^{2} v=c^{2} \partial_{x}^{2} v-r v
$$

where $\mathrm{c}^{2}=1$ and $\mathrm{r}=1$, with the boundary and initial conditions are $v(0, t)=0, \quad v(\pi, t)=0, \quad t>0$ $v(x, 0)=V(x) \quad, \quad \partial_{t} v(x, 0)=0,0 \leq x \leq \pi$ where $\mathrm{V}(\mathrm{x})$ is piecewise smooth. 
To solve this example we impose two forms of $\mathrm{V}(\mathrm{x})$, the sine function

$$
\mathrm{V}_{1}(\mathrm{x}, 0)=\sin (10 \pi \mathrm{x}(\mathrm{x}+1)) \text {, }
$$

and the polynomial function

$$
\mathrm{V}_{2}(\mathrm{x}, 0)=21 \mathrm{x}^{2}-13.4 \mathrm{x}+2.4
$$

then take the space step size $\mathrm{h}=0.3143$ and time step size $\mathrm{k}=0.5$, where $\mathrm{n}=11$, and $\mathrm{m}=21$. The solutions are given in figure (1) and (2).

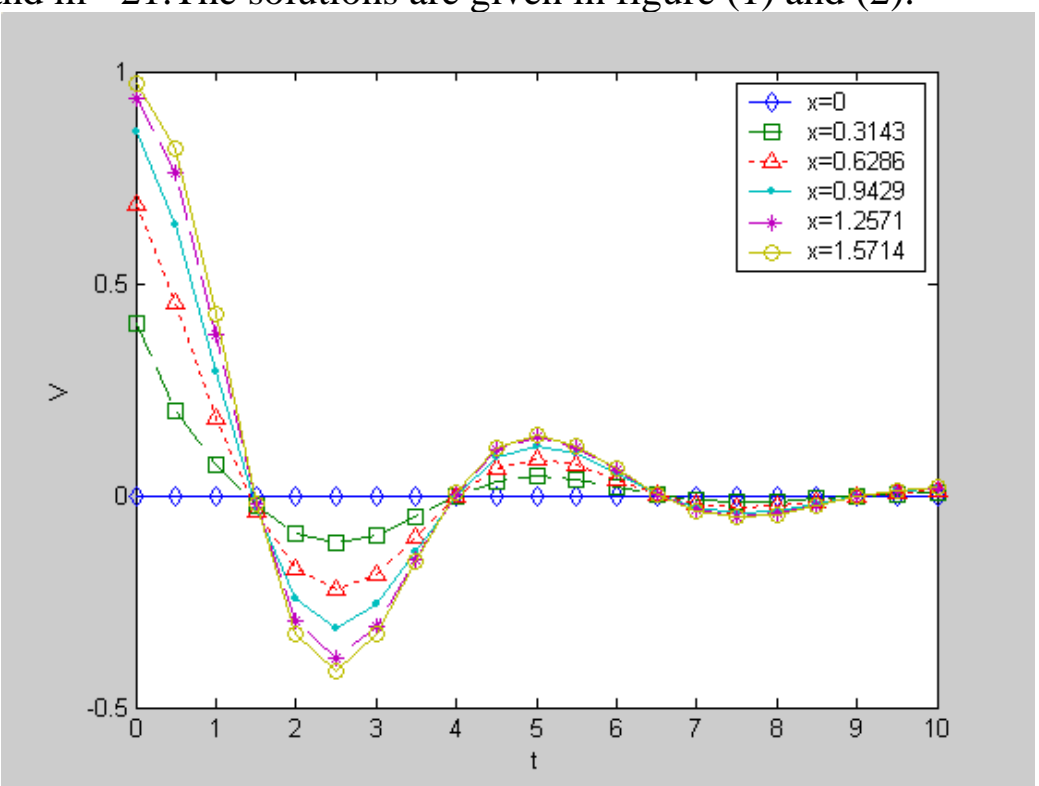

Figure (1): Numerical solutions of the example (1) using the initial condition $\mathrm{V}_{1}(\mathrm{x}, 0)=\sin (10 \pi \mathrm{x}(\mathrm{x}+1))$

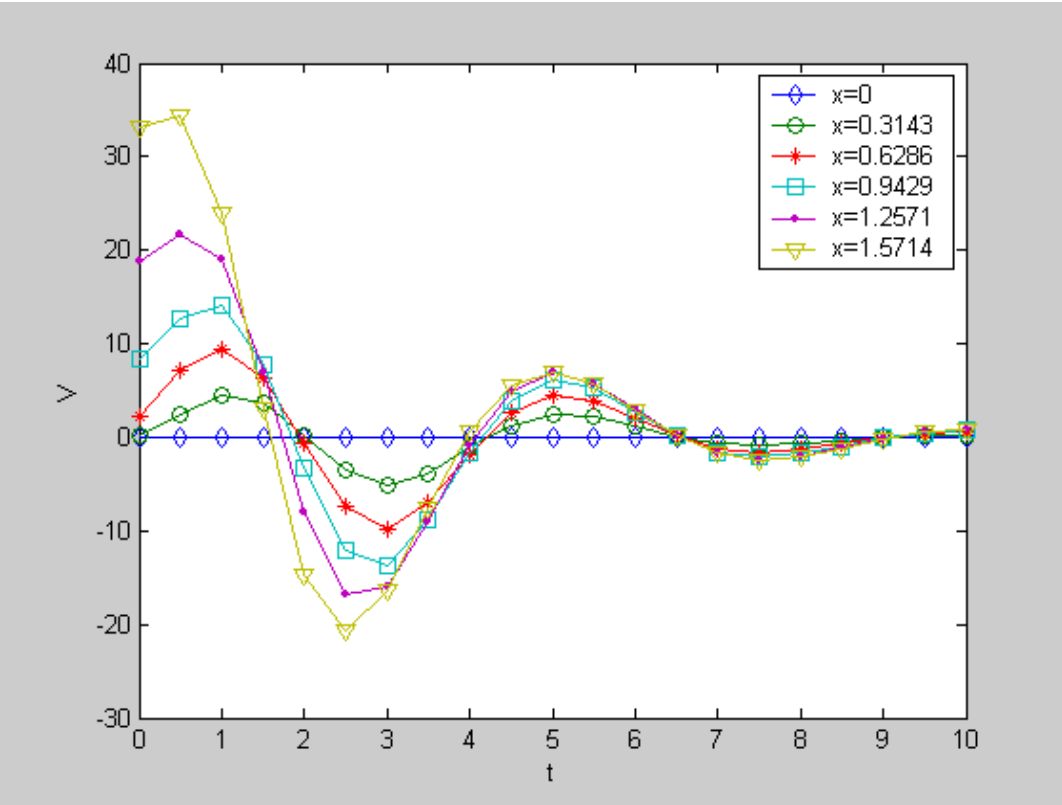

Figure (2): Numerical solutions of the example (1) using the initial condition $\mathrm{V}_{2}(\mathrm{x}, 0)=21 \mathrm{x}^{2}-13.4 \mathrm{x}+2.4$ 
Table (1): numerical solutions using the implicit finite difference method at $\mathrm{t}=0.9429$ of the example $(1)$

\begin{tabular}{|l|l|l|}
\hline The values of $\mathbf{t}$ & $\begin{array}{l}\text { Solution (1) with } \\
\text { the initial } \\
\text { condition } \mathbf{V}_{\mathbf{1}}(\mathbf{x}, \mathbf{0})\end{array}$ & $\begin{array}{c}\text { Solution (2) with } \\
\text { the initial condition } \\
\mathbf{V}_{\mathbf{2}}(\mathbf{x , 0}, \mathbf{0})\end{array}$ \\
\hline 0 & .8558701 & 8.435409 \\
0.5000 & .6377119 & 12.62647 \\
1.0000 & .2921410 & 14.08372 \\
1.5000 & $-.3158034 \mathrm{e}-1$ & 7.812533 \\
2.0000 & -.2451307 & -3.287807 \\
2.5000 & -.3149860 & -12.02026 \\
3.0000 & -.2587071 & -13.64413 \\
3.5000 & -.1319557 & -8.829426 \\
4.0000 & $-.2699218 \mathrm{e}-3$ & -1.649570 \\
4.5000 & $.8874284 \mathrm{e}-1$ & 3.934614 \\
5.0000 & .1180779 & 6.105594 \\
5.5000 & $.9752016 \mathrm{e}-1$ & 5.209351 \\
6.0000 & $.5090318 \mathrm{e}-1$ & 2.716023 \\
6.5000 & $.2839250 \mathrm{e}-2$ & .1437973 \\
7.0000 & $-.3001029 \mathrm{e}-1$ & -1.554242 \\
7.5000 & $-.4180047 \mathrm{e}-1$ & -2.109822 \\
8.0000 & $-.3571276 \mathrm{e}-1$ & -1.754360 \\
8.5000 & $-.1979325 \mathrm{e}-1$ & -.9387796 \\
9.0000 & $-.2628169 \mathrm{e}-2$ & $-.9779943 \mathrm{e}-1$ \\
9.5000 & $.9674528 \mathrm{e}-2$ & .4843633 \\
10.0000 & $.1466398 \mathrm{e}-1$ & .7083982 \\
\hline
\end{tabular}

From table (1) we can see that the numerical solutions which are obtained by the implicit finite difference method at the point $\mathrm{x}=0.9429$ are converged ( which is clear from figure (1) and figure (2)). We compared between two solutions (1) and (2) by taking two initial conditions and we obtained the solution (1) as the best convergence. But, the solution (2) will start with a blowing-up which makes a slight difference from solution (1) in converging at each time step. Therefore, the solution of this example is increasing and is decreasing then it vanishes and converges to zero at each space step size.

\section{Example (2):}

we consider a nonlinear partial differential equation (NKG) [1]

$$
\partial_{t t} v-\partial_{x x} v+v^{2}-v v_{x}=0 \quad, \quad 0 \leq x \leq 1, \quad t>0
$$

The boundary conditions and initial conditions posed are

$$
\begin{aligned}
& v(0, t)=0, \quad v(1, t)=1, \quad t \geq 0 \\
& v(x, 0)=v_{t}(x, 0)=e^{x}, 0 \leq x \leq 1
\end{aligned}
$$


We take the space step size $\mathrm{h}=0.1$ and time step size $\mathrm{k}=0.05$, where $\mathrm{n}=11$, and $\mathrm{m}=21$. The solutions are given in figure (3).

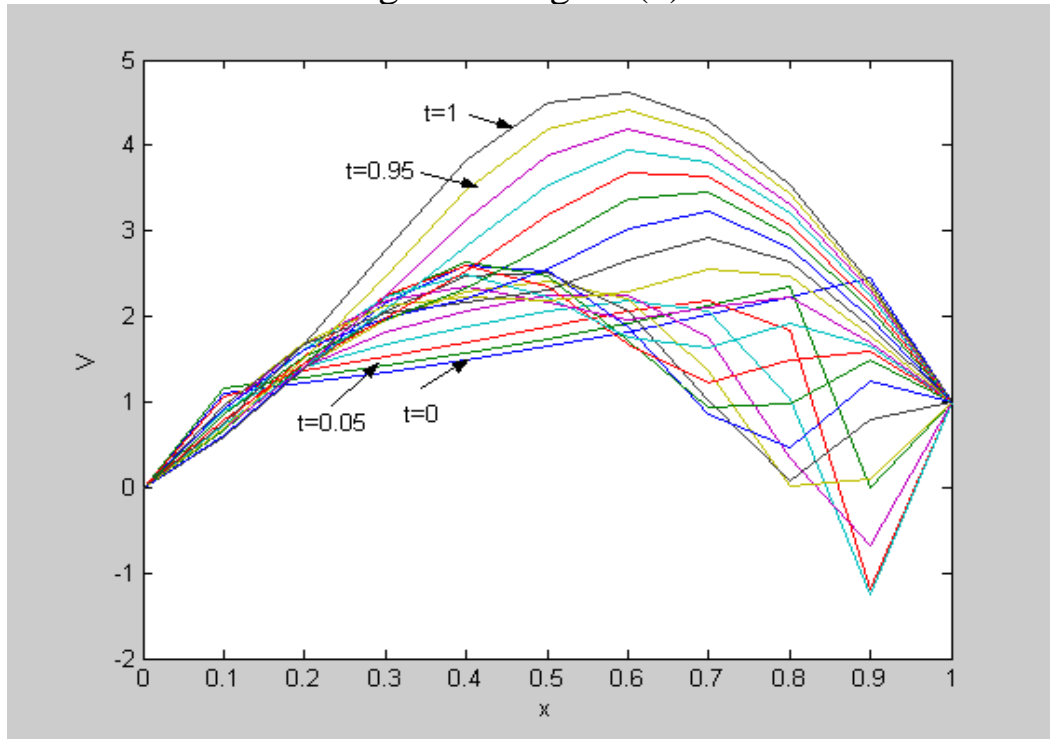

Figure (3): Numerical solutions of the example (2) using the implicit finite difference method

If we impose the initial condition of the form $v(x, 0)=\sin (x), 0 \leq x \leq 1$, then we obtain figure (4)

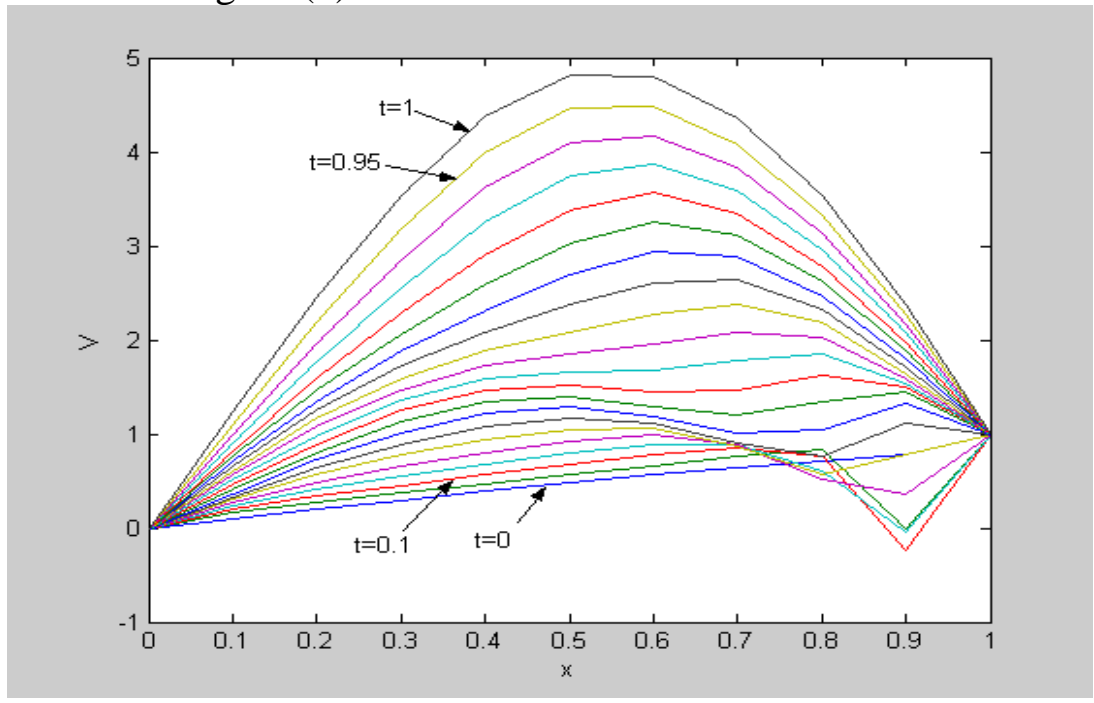

Figure (4): Numerical solutions of the example (2) using the implicit finite difference method with $\mathrm{v}(\mathrm{x}, 0)=\sin (\mathrm{x})$ 
Table (2): numerical solutions using the implicit finite difference method at $\mathrm{t}=0.35$ of the example (2)

\begin{tabular}{|l|l|l|}
\hline The values of $\mathbf{x}$ & $\begin{array}{c}\text { Solution (1) with the } \\
\text { initial condition } \\
\mathbf{e x p}(\mathbf{x})\end{array}$ & $\begin{array}{c}\text { Solution (2) with the } \\
\text { initial condition } \\
\mathbf{s i n}(\mathbf{x})\end{array}$ \\
\hline 0 & 0 & 0 \\
0.1 & .6057965 & 0.3661 \\
0.2 & 1.401828 & 0.7196 \\
0.3 & 2.159058 & 1.0142 \\
0.4 & 2.590769 & 1.2141 \\
0.5 & 2.532223 & 1.2828 \\
0.6 & 1.877799 & 1.1880 \\
0.7 & .8556628 & 1.0083 \\
0.8 & .4664556 & 1.0429 \\
0.9 & 1.246132 & 1.3248 \\
1.0 & 1.0000 & 1.0000 \\
\hline
\end{tabular}

Table (2) compares between two solutions by taking two initial conditions as $\exp (\mathrm{x})$ and $\sin (\mathrm{x})$ functions, respectively. It is clear from the two figures (3) and (4) that the $\sin (\mathrm{x})$ function converges uniformly faster than the $\exp (\mathrm{x})$ function.

\section{5- Conclusions}

In this study, the solution of the matrix equation for one-dimensional Klein-Gordon equation exists. It is clear from numerical computations that the solutions are stable and convergent when the sine function is used as an initial condition. 


\section{REFERENCES}

[1] Bektas, M.;H.Bulut and M.Ergut, "Geometric Interpretations of the Klein-Gordon Equation and solution of the equation by the Adomain Decomposition Method", F.U. Fen ve Muhendislik Bilimleri Dergisi, 16(3),411-423, 2004.

[2] Delort, Jean-Marc, J. Szeftel," Almost global solutions for non Hamiltonian semi-linear Klein-Gordon equations on compact revolution hypersurfaces", Journ'ees ' Equations aux d'eriv'ees partielles Forges-les-Eaux, 6 juin-10 juin , 2005.

[3] Landa , P.S., "Nonlinear Oscillations and Waves in Dynamical Systems", Kluwer Academic Publishers , 1996

[4] Mugnai, Dimitri, "Coupled Klein-Gordon and Born-Infled type equations: looking for solitary waves", Article submitted to Royal Society, 2002.

[5] Nicolas, Jean-Philippe, "A nonlinear Klein-Gordon equation on Kerr metrics", J. Math Pures et Appliquées, 81 ,9, pp. 885-914 ,2002.

[6] www//amath.colorado.edu/grad/prelims/Pde/2005a.pdf Partial Differential equations, 2005. 